\title{
Cytotoxic proteins of human placenta
}

\author{
S. Skripkin ${ }^{1 *}$, E. Burkova ${ }^{2}$, G. Nevinsky ${ }^{1,2}$ \\ ${ }^{1}$ Novosibirsk State University, Novosibirsk, Russia \\ ${ }^{2}$ Institute of Chemical Biology and Fundamental Medicine, Novosibirsk, Russia \\ *e-mail: skripkin.stan@gmail.com
}

Key words: human placenta, proteins of the placenta, protein complexes, inhibition of cell proliferation, apoptosis

Motivation and Aim: Numerous biological functions of the placenta are carried out by various oligomeric structures and complexes. The activity of protein complexes can significantly differ from the activity of individual proteins. Previously, a highly stable soluble protein complex of the human placenta was isolated, with a molecular mass reaching $1000 \mathrm{kDa}$. It was found that the complex includes human serum albumin, transferrin, IgG, annexin and other proteins. This complex exhibited DNA-hydrolyzing and catalase activity $[1,2]$. The purpose of this work is to study proteins and protein complexes of the human placenta, to study their composition and properties.

Methods and Algorithms: The placentas of ten healthy women was used in the work after normal physiological parturition. The proteins of the placenta extract were separated by gel filtration on a column with Sepharose 4B sorbent. The protein fractions were examined using an MTT test and flow cytofluorometry with treatment with Annexin B and propidium iodide. SDS electrophoresis was used to evaluate the molecular masses of proteins.

Results: After gel filtration of protein extracts in all cases two protein peaks were observed. The MTT test of the obtained fractions showed that some fractions of the second peak of the gel filtration of the human placenta extract inhibit the proliferation of cells of both the cancer line MCF-7 and normal WI-38. Flow cytofluorometry with treatment with Annexin $\mathrm{V}$ and propidium iodide revealed that cytotoxically active fractions of the human placenta cause cell death by the mechanism of apoptosis. Using SDS electrophoresis, molecular weights of proteins of cytotoxic fractions of human placenta have been determined. In the main in the factions are proteins with molecular masses of 64.7, 51.2, 24.8 and $14.8 \mathrm{kDa}$, minor - 179.4, 44 and $33.1 \mathrm{kDa}$.

Conclusion: The study of proteins and protein complexes of the human placenta will allow a deeper understanding of the functional characteristics of certain proteins, as well as the mechanisms of the functioning of the placenta.

Acknowledgements: The work is supported by the project No. VI.62.1.5. The program of FSI SB RAS VI.62.1 (0309-2018-0003) and the RFBR grant No. 16-04-00609.

\section{References}

1. Burkova E.E., Dmitrenok P.S., Sedykh S.E., Buneva V.N., Soboleva S.E., Nevinsky G.A. (2014) Extremely Stable Soluble High Molecular Mass Multi-Protein Complex with DNase Activity in Human Placental Tissue. PLOS One. DOI 10.1371/journal.pone.0111234.

2. Burkova E.E., Nevinsky G.A. (2016) Very stable high molecular mass multi-protein complex from human placenta. FEBS. 283(1):211. 